\title{
Substances bioactives élaborées par des cyanobactéries isolées de certains écosystèmes aquatiques marocains
}

\section{Khalid OUFDOU* et Brahim OUDRA}

Equipe de Microbiologie et Toxicologie Environnementales, Laboratoire de Biologie et de Biotechnologie des Microorganismes, Faculté des Sciences Semlalia Marrakech, B.P. 2390, Marrakech 40 000, Maroc.

*Correspondance,courriel : oufdov@ucam.ac.ma

\section{Résumé}

Durant la saison chaude, plusieurs écosystèmes aquatiques (lacs, étangs, rivières, ...) connaissent une croissance importante des cyanobactéries conduisant à la formation des efflorescences (blooms). Les cyanobactéries sont capables de produire une multitude de substances antimicrobiennes: antibactériennes, algicides, antifongique, antivirales et même des substances toxiques.

L'objectif de ce travail constitue en l'étude des effets des substances bioactives produites par 10 souches de cyanobactéries isolées de certains écosystèmes aquatiques marocains. Ainsi, nous avons purifié et identifié les souches cyanobactéries isolées et nous avons testé l'activité de leurs substances extracellulaires et intracellulaires sur des micro-organismes d'intérêt sanitaire. Nous avons aussi évalué leur toxicité par le biotest souris.

L'identification des 10 souches de cyanobactéries étudiées a montré qu'elles appartiennent à 3 familles à savoir les Oscillatoriaceae, les Nostocaceae et les Chroococcaceae.

L'étude des effets des substances intracellulaires produites par ces souches de cyanobactéries, a montré que les extraits aqueux de ces substances ne donnent pas généralement une importante activité. Par contre, ces substances extraites par le méthanol inhibent la croissance de Micrococcus luteus et de Bacillus cereus. Les souches de cyanobactéries responsables de cette activité sont: Lyngbya sp., Pseudanabaena mucicola, Oscillatoria chlorina, Phormidium sp., Nostoc muscorum, Oscillatoria putrida et Microcystis aeruginosa. 
Les substances extracellulaires produites par les cyanobactéries étudiées ont aussi une activité antibactérienne vis-à-vis de $M$. luteus, Bacillus subtilis, Serratia marcescens, B. cereus, Escherichia coli0128B12 et Staphylococcus aureus.

Le biotest souris a permis de montrer que certaines cyanobactéries étudiées entraînent une mortalité des souris auxquelles sont injectées les cyanotoxines; ce qui montre que certaines souches de cyanobactéries produisent également des substances toxiques.

Mots-clés : Activité antimicrobienne, cyanobactéries, écosystèmes aquatiques, substances extracellulaires, substances intracellulaires, toxicité.

\section{Abstract \\ Bioactive compounds produced by Cyanobacteria isolated from some aquatic ecosystems in Morocco}

During hot periods, several aquatic ecosystems are characterized by blooms of cyanobacteria. The cyanobacteria are able to produce bioactive products which have a broad spectrum of properties such as antibacterial, antifungal, antiviral, algicidal or toxic activities

The aim of the present study is the screening of bioactive compounds produced by 10 strains of cyanobacteria isolated from some aquatic ecosystems.

We have purified and identified cyanobacterial strains and tested the activity of their extracellular and endocellular products against some microorganisms of sanitary interest. We have also tested their toxicity using mouse bioassays.

The identification of 10 strains of cyanobacteria isolated showed that they belong to 3 families: Oscillatoriaceae, les Nostocaceae et les Chroococcaceae.

Aqueous extracts of endocellular products didn't present any important activity. Whereas, methanolic extracts of endocellular products presented antibacterial activity against $M$. luteus and $B$. cereus. $O$. putrida (SI) and M. aeruginosa (S4) presented antibacterial activity against $M$. luteus whereas Phormidium sp. (S6) and $P$. mucicola (S3) have an activity against $M$. luteus and $B$. cereus. The strains of cyanobacteria which caused this activity were: Lyngbya sp., Pseudanabaena mucicola, Oscillatoria chlorina, Phormidium sp., Nostoc muscorum, Osicllatoria putrida, et Microcystis aeruginosa.

The extracellular products of cyanobacterial strains had also antibacterial activity on M. Iuteus, Bacillus subtilis, Serratia marcescens, B. cereus, Escherichia coli0128B12 et Staphylococcus aureus. 
The research of toxic substances released by the cyanobacterial strains revealed that some cyanobacteria presented toxic activity against mouse. In contrast, 0 . putrida (SI) which has the most antibacterial activity did not show any toxic activity.

Keywords : antimicrobial activity, aquatic ecosystems, Cyanobacteria, extracellular products, intracellular products, toxicity.

\section{Introduction}

Les cyanobactéries appelées autrefois algues bleues vertes, sont des procaryotes photosynthétiques qui se présentent sous forme de cellules isolées, en amas ou en filaments. Ces micro-organismes peuplent une grande variété de milieux aquatiques: les eaux douces, courantes (rivières, torrents), les eaux stagnantes (étangs, lacs), les eaux usées et les eaux littorales [1]. Dans ces milieux aquatiques, les cyanobactéries sont souvent l'origine d'intoxications mortelles de troupeaux d'animaux domestiques ou sauvages, de poissons, d'oiseaux et même de l'Homme. En effet, les cyanobactéries sont capables de produire des substances possédant des propriétés toxiques à savoir l'hépatotoxicité, la neurotoxicité, la dermatotoxicité et les syndromes gastro-intestinaux [2-4].

En revanche, plusieurs travaux ont montré que les cyanobactéries constituent une nouvelle source de composés bioactifs [5-7]. En effet, les cyanobactéries sont capables de produire une multitude de substances antimicrobiennes; certaines d'entre elles possèdent des effets algicides $[8,9]$ permettant ainsi aux cyanobactéries d'être plus compétitives en présence avec d'autres algues. D'autres substances ont une activité antifongique $[10,11]$. Les cyanobactéries produisent aussi des substances antibactériennes $[12,13]$ et même antivirales $[5,7]$.

Les cyanobactéries présentent un pouvoir important d'adaptation aux changements environnants et un taux de croissance rapide. Ainsi, elles ont la possibilité de développer une grande masse cellulaire [14]. L'étude des substances bioactives produites par ces micro-organismes mérite d'être étudiée. En effet, peu de travaux ont été réalisés sur la production de substances bioactives élaborées par des souches de cyanobactéries isolées des écosystèmes aquatiques marocains.

Ainsi, les objectifs de cette étude sont :

- Isoler, purifier et identifier des cyanobactéries à partir de certains écosystèmes aquatiques marocains.

- Déterminer les activités antibactériennes et antifongiques des substances bioactives élaborées par ces cyanobactéries. 
- Etudier la production de substances bioactives toxiques par ces souches de cyanobactéries en réalisant le biotest souris.

\section{Matériel et méthodes}

\section{2-1. Sites d'étude}

Nous avons travaillé avec des souches de cyanobactéries isolées des 4 écosystèmes aquatiques suivants :

- Le lac-réservoir Lalla Takerkoust: il est situé à $35 \mathrm{Km}$ au Sud-Ouest de Marrakech. II a une longueur de $7 \mathrm{Km}$, une largeur de $1 \mathrm{Km}$ et une profondeur maximale de $25 \mathrm{~m}$. Ce réservoir est utilisé surtout pour l'irrigation et pour la production d'électricité.

- La région d'Oukaimden se trouve à une altitude de $2600 \mathrm{~m}$ dans le grand Atlas. Elle est située à $70 \mathrm{Km}$ au Nord de Marrakech. Cette région est caractérisée par un climat méditerranéen de haute montagne.

- Le lac-réservoir Oued Mellah est situé $270 \mathrm{~km}$ au nord-ouest de Marrakech, il a une profondeur maximale de $9 \mathrm{~m}$. Il est caractérisé par un climat méditerranéen semi-aride à hiver chaud. II est utilisé pour l'irrigation, la pêche et pour les activités de loisirs.

- La région d'Ourika se situe dans la partie centrale du Haut-atlas de Marrakech à une quarantaine de kilomètres au Sud de cette ville. Son climat est typiquement méditerranéen à influence semi-continentale.

\section{2-2. Méthodes d'isolement, de purification et d'identification des cyanobactéries}

Des échantillons de cyanobactéries isolées des écosystèmes aquatiques précités sont mis en cultures dans des boites de pétri contenant le milieu $B G_{13}$ et/ou le milieu $Z_{8}$. L'incubation des cultures algales est réalisée dans une chambre thermostatée à une température de $25 \pm 1^{\circ} \mathrm{C}$. Elles sont éclairées par 3 lampes blanches fluorescentes de $40 \mathrm{~W}$ à une intensité de $50 \mu \mathrm{mol} / \mathrm{m}^{2} / \mathrm{S}$. La photopériode est de l'ordre de $16 \mathrm{~h}$ de lumière et $8 \mathrm{~h}$ d'obscurité.

Les repiquages successifs sur les milieux gélosés $\left(B G_{13}\right.$ ou $\left.Z_{8}\right)$ ont permis d'obtenir des cultures unialgales. Afin d'obtenir une grande masse cellulaire qui sera utilisée dans les étapes suivantes du travail, les cultures unialgales sont transférées dans 
des flacons contenant les milieux liquides $\left(B G_{13}\right.$ et/ou $\left.Z_{8}\right)$ et aérés par l'air ambiante stérile apportée à l'aide de pompes à air.

L'identification des espèces de cyanobactéries est basée sur l'étude des caractères morphologiques en utilisant le microscope photonique $[15,16]$.

\section{2-3. Tests d'activité antibactérienne et antifongique}

Les substances intracellulaires ainsi que les substances extracellulaires produites par les cyanobactéries sont testées vis-à-vis de certaines bactéries d'intérêt sanitaire (Micrococcus luteus, Bacillus subtilis, Bacillus cereus, Escherichia coli 0128B12, Serratia marcescens, Staphylococcus aureus et Salmonella typhimurium ATC( 43971 ) et sur la levure Candida albicans. (es tests sont réalisés en milieu liquide et en milieu solide (technique des disques, techniques des puits et technique des cylindres d'agar). Les souches non référencées utilisées dans le cadre de ce travail sont de nature clinique.

\section{2-3-1. Effet des substances extracellulaires: Technique des cylindres d'agar}

Pour tester l'effet des substances extracellulaire produites par les cyanobactéries nous avons utilisé la technique des cylindres d'agar :

Les souches de cyanobactéries sont ensemencées sur des boites de pétri contenant le milieu $Z_{8}$ solide de façon à obtenir un tapis cyanobactérien. Par la suite, nous prélevons des cylindres d'agar d'environ $9 \mathrm{~mm}$ de diamètre et nous les déposons sur des boites de pétri contenant des milieux gélosés (gélose nutritive pour les bactéries et le milieu de Sabouraud pour la levure) et ensemencées par les micro-organismes tests par la technique d'inondation. Les boites de pétri sont ensuite laissées pendant 2 heures à $4^{\circ} \mathrm{C}$ (pour permettre la diffusion des substances bioactives) puis incubées à $30^{\circ} \mathrm{C}$ pendant $24 \mathrm{~h}$. Nous mesurons alors le diamètre des zones d'inhibition.

\section{2-3-2. Effet des substances intracellulaires}

\section{a-Préparation des extraits aqueux}

Les cellules des cultures en batch des souches de cyanobactéries sont récoltées dans le culot après centrifugation à $7000 \mathrm{rpm}$ pendant 15 minutes. $200 \mathrm{mg}$ de ce matériel cyanobactérien subit un gel-dégel suivi d'une sonification (100 khz pendant 5 minutes) afin d'éclater les cellules. Après nous réalisons une centrifugation (5000 
rpm pendant 30 minutes) pour éliminer les débris de cyanobactéries. Le surnageant contient ainsi les substances intracellulaires dont l'effet est testé sur les microorganismes tests par la technique des puits. $5 \mathrm{ml}$ du milieu gélosé spécifique pour la croissance du micro-organisme test sont répartis en boites de pétri. Après solidification, $10 \mathrm{ml}$ du même milieu sont ajoutés puis nous déposons des billes de verre stériles. Après solidification, les billes sont enlevées, elles laissent alors apparaître des puits de volume identique. Nous inondons les boites avec le microorganisme test. Les puits déjà préparés sont prêts à recevoir un volume de $100 \mu \mathrm{l}$ de suspension à tester. Les boites de pétri sont ensuite laissées pendant $2 \mathrm{~h}$ à $4^{\circ} \mathrm{C}$ avant leur incubation à $30^{\circ} \mathrm{C}$ pendant $24 \mathrm{~h}$, puis nous mesurons le diamètre de la zone d'inhibition.

\section{b- Préparation des extraits méthanoliques}

La préparation de l'extrait méthanolique est réalisée trois fois successives par du méthanol à 100\%. $200 \mathrm{mg}$ du matériel cyanobactérien sont extraits trois fois par 100 $\mathrm{ml}$ de méthanol. Chaque fois, les extraits sont centrifugés à $4000 \mathrm{~g}\left(10 \mathrm{~min}, 4^{\circ} \mathrm{C}\right)$, les surnageants sont retenus alors que le culot est réextrait à nouveau. Les trois extraits sont finalement recombinés et concentrés au rotavapeur, par la suite nous testons l'activité sur les micro-organismes étudiés par la technique des disques. Les disques de papier d'environ $6 \mathrm{~mm}$ de diamètre reçoivent $100 \mu \mathrm{l}$ d'une solution antimicrobienne à tester (extrait méthanolique des substances intracellulaires). Après leur séchage, les disques sont déposés sur des boites de pétri contenant des milieux gélosés et ensemencés par les micro-organismes tests. Les boites de pétri sont laissées pendant 2 heures à $4^{\circ} \mathrm{C}$ puis incubées à $30^{\circ} \mathrm{C}$ pendant $24 \mathrm{~h}$. Nous mesurons alors le diamètre de la zone d'inhibition.

\section{2-4. Test de toxicité}

Les cellules des cultures en batch des souches de cyanobactéries sont récoltées après centrifugation à $7000 \mathrm{rpm}$ pendant 15 minutes. $200 \mathrm{mg}$ de ce matériel algal est repris dans $4 \mathrm{ml}$ d'eau physiologique. L'ensemble a subit une sonification (100 khz pendant 5 minutes). Nous réalisons ensuite une centrifugation (5000 rpm pendant 30 minutes) pour éliminer les débris de cyanobactéries. Un $\mathrm{ml}$ de la solution obtenue est injecté intrapéritonéalement à des souris de laboratoire ayant un poids entre 20 et $22 \mathrm{~g}$. Les souris témoins sont injectées par de l'eau physiologique stérile. Une cyanobactérie est considérée toxique si la souris meurt dans les 24 heures qui suivent l'injection. 


\section{Résultats et discussion}

\section{3-1. Identification des espèces de cyanobactéries}

La culture des échantillons de cyanobactéries sur le milieu solide $B G_{13}$ et $Z_{8}$ et les repiquages successifs ont permis de purifier 10 souches de cyanobactéries (TableaU $I$ et Figure $\eta$.

Tableau 1 : Les espèces de cyanobactéries après identification.

\begin{tabular}{|l|l|l|l|}
\hline Souches & Espèces & Familles & Lieux d'isolement \\
\hline S1 & Oscillatoria putrida & Oscillatoriaceae & Lac Lalla Takerkoust \\
\hline S2 & $\begin{array}{l}\text { Pseudanabaena } \\
\text { galeata }\end{array}$ & Oscillatoriaceae & Lac Lalla Takerkoust \\
\hline S3 & Lyngbya sp. & Oscillatoriaceae & Oued Ourika \\
\hline S4 & $\begin{array}{l}\text { Pseudanabaena } \\
\text { mucicola }\end{array}$ & Oscillatoriaceae & Lac Lalla Takerkoust \\
\hline S5 & Oscillatoria chlorina & Oscillatoriaceae & Oued Mellah \\
\hline S6 & Microcystis aeruginosa & Chroococcaceae & Lac Lalla Takerkoust \\
\hline S7 & $\begin{array}{l}\text { Anabaena } \\
\text { aphanizomenoides }\end{array}$ & Nostocaceae & Oued Mellah \\
\hline S8 & Lyngbya sordida & Oscillatoriaceae & Lac Lalla Takerkoust \\
\hline S9 & Phormidium sp. & Oscillatoriaceae & Lac Lalla Takerkoust \\
\hline S10 & Nostoc muscorum & Nostocaceae & Région Oukaimden \\
\hline
\end{tabular}

Ces espèces appartiennent à 3 familles de cyanobactéries à savoir les Oscillatoriaceae, les Nostocaceae et les Chroococcaceae, et elles représentent 7 genres qui sont caractérisés par [15, 16] :

\section{*Famille des Oscillatoriaceae :}

- Oscillatoria: est une cyanobactérie filamenteuse dont le trichome est libre, solitaire et dépourvue de gaine. Le mouvement et le déplacement hélicoïdal de l'apex sont caractéristiques de ce genre.

- Pseudanabaena: cyanobactérie filamenteuse dont les trichomes sont solitaires, mobiles et sans gaine. Les cellules sont distantes les unes des autres et réunies par un pont gélatineux. II n'y a ni akinètes ni hétérocystes. 
- Lyngbya: une cyanobactérie filamenteuse dont les filaments sont généralement solitaires et possédant une gaine caractéristique.

- Phormidium: Elle a les mêmes caractéristiques que Lyngbya sauf qu'elle a une gaine peu épaisse.

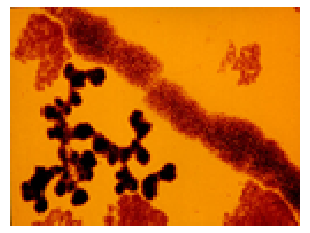

S6: Marogitis aenginosa $(X 400)$

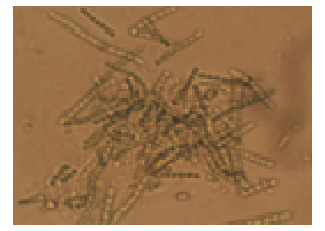

S4: Pseudanabaena mucicola $(x 1000)$

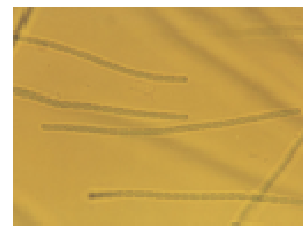

S2: Pseudamabaena galeata $(X 400)$

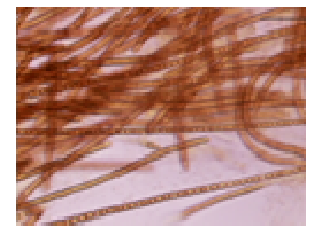

S9: Phormidium sp. $(X 1000)$

\section{Lac du barrage Lalla Takerkoust}

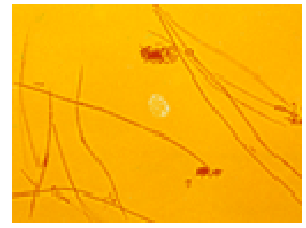

S7: Anabaena aphanizomenoides $(T$ 400)

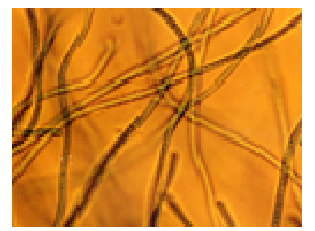

S5: Oscillatoria chlorina $(X$ 1000)

Lac du barrage Oned Mellah

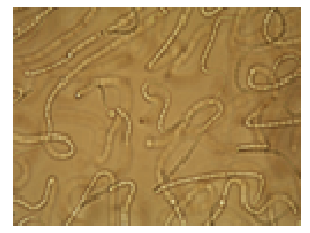

510: Mostoc muscorum $(x 400)$

Région

d'oukaimden

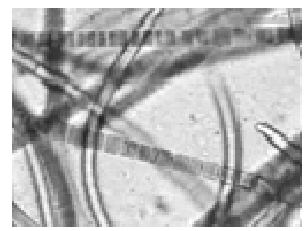

S3: Lyngbya sp. $(\pi 1000)$

Oued Ourika

Figure 1 : Photos de certaines cyanobactéries classées selon les sites d'échantillonnage.

\section{* Famille des Nostocaceae :}

- Anabaena: une cyanobactérie dont les trichomes sont simples, réguliers et d'égale épaisseur. Elle présente des hétérocystes intercalaires et on note l'absence de gaine. - Nostoc: une cyanobactérie dont les thalles sont gélatineux et contiennent, dans une gelée commune, des trichomes enchevêtrés avec des hétérocystes intercalaires.

\section{* Famille des Chroococcaceae :}

- Microcystis: Elle est une cyanobactérie unicellulaire dont les colonies sont sphériques, groupées dans une enveloppe et qui sont flottantes à l'aide des vacuoles gazeuses. 
Les Oscillatoriaceae représentent $70 \%$ des souches isolées. La dominance des cyanobactéries de la famille des Oscillatoriaceae au sein de la communauté phytoplanctonique des lacs peu profonds et des étangs a été rapportée [17]. Leur dominance peut parfois persister toute l'année dans les plans d'eau turbides et lorsque l'hiver n'est pas très froid [17].

Plusieurs espèces de cette famille ont été inventoriées au Maroc dont Pseudanabaena galeata, Pseudanabaena mucicola, Oscillatoria chlorina [18] et Lyngbyasp. [19, 20].

La classe des Chroococcaceae (10\% des souches isolées) est représentée ici par le genre Microcystis qui inclut les espèces les plus étudiées et géographiquement les plus distribuées [19]. Au Maroc, plusieurs espèces ont été inventoriées, mais l'espèce la plus commune est Microcystis aeruginosa [19] qui est responsable de la formation de blooms au Lac Lalla Takerkoust [18].

Les Nostocaceae (20\% des souches isolées) sont représentées par Anabaena aphanizomenoides et Nostoc muscorum. Anabaena est une espèce cosmopolite et elle est souvent incriminée dans la formation de blooms toxiques [20]. Plusieurs espèces de ce genre ont été inventoriées au Maroc dont Anabaena aphanizomenoides occasionnant des blooms toxiques au Lac Oued Mellah [21]. L'autre espèce de cette famille Nostoc muscorum est souvent rencontrée dans la région d'Oukaimden [22].

\section{3-2. Tests d'activité antibactérienne et antifongique}

Nous avons réalisé un screening des substances bioactives élaborées par ces cyanobactéries vis-à-vis des micro-organismes d'intérêt sanitaire. Pour cela les substances intracellulaires et extracellulaires de ces 10 souches de cyanobactéries sont testées sur 8 germes tests dont 7 bactéries et une levure. En effet, les extraits aqueux et méthanoliques des substances intracellulaires ainsi que les substances extracellulaires sont testés vis-à-vis des bactéries à Gram négatif à savoir $E$. coli, $S$. marcescens et $S$. typhimurium, sur des bactéries à Gram positif à savoir M. Iuteus, $S$. aureus, B. cereus, B. subtilis et sur la levure C. albicans.

Parmi les 10 souches de cyanobactéries, sept $(70 \%)$ sont actives et elles ont présenté au moins une réaction positive (RP) vis-à-vis des germes tests étudiés, alors que les trois restantes à savoir $P$. galeata (S2), A. aphanizomenoides (ST) et $L$. sordida (S8), sont non actives (Figure 2). 


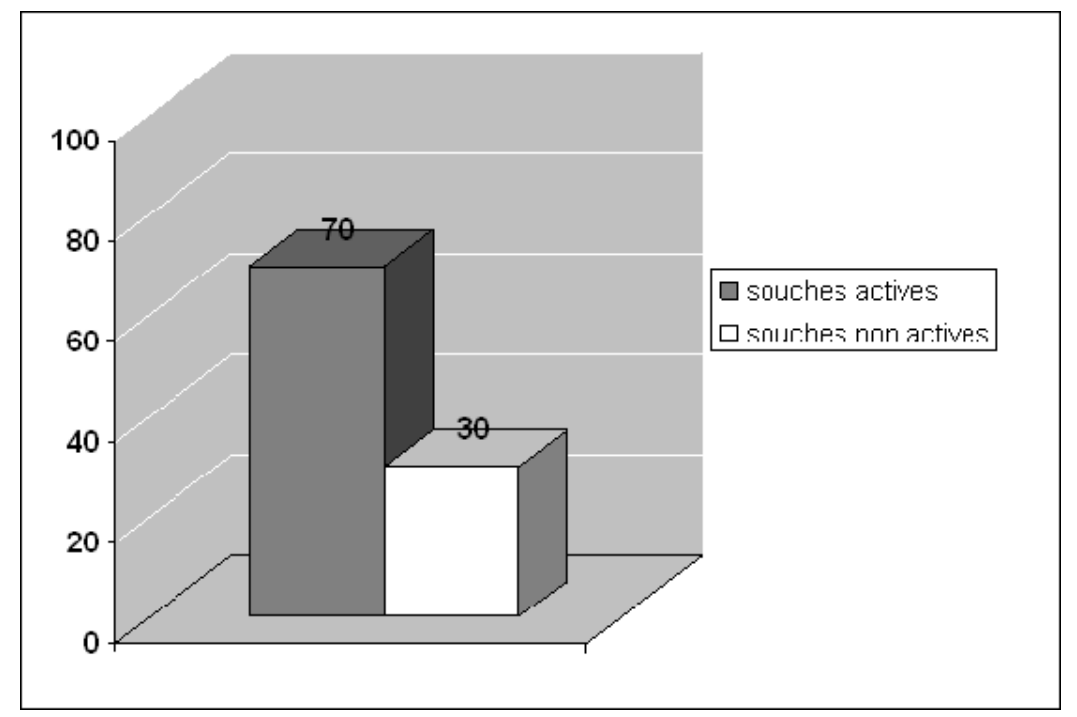

Figure 2 : Pourcentage des souches de cyanobactéries actives.

Cette activité est due à $35 \%$ des substances intracellulaires et à $25 \%$ de substances extracellulaires, alors que $15 \%$ de substances intracellulaires et $25 \%$ de substances extracellulaires n'ont présenté aucun effet (Figure 3).

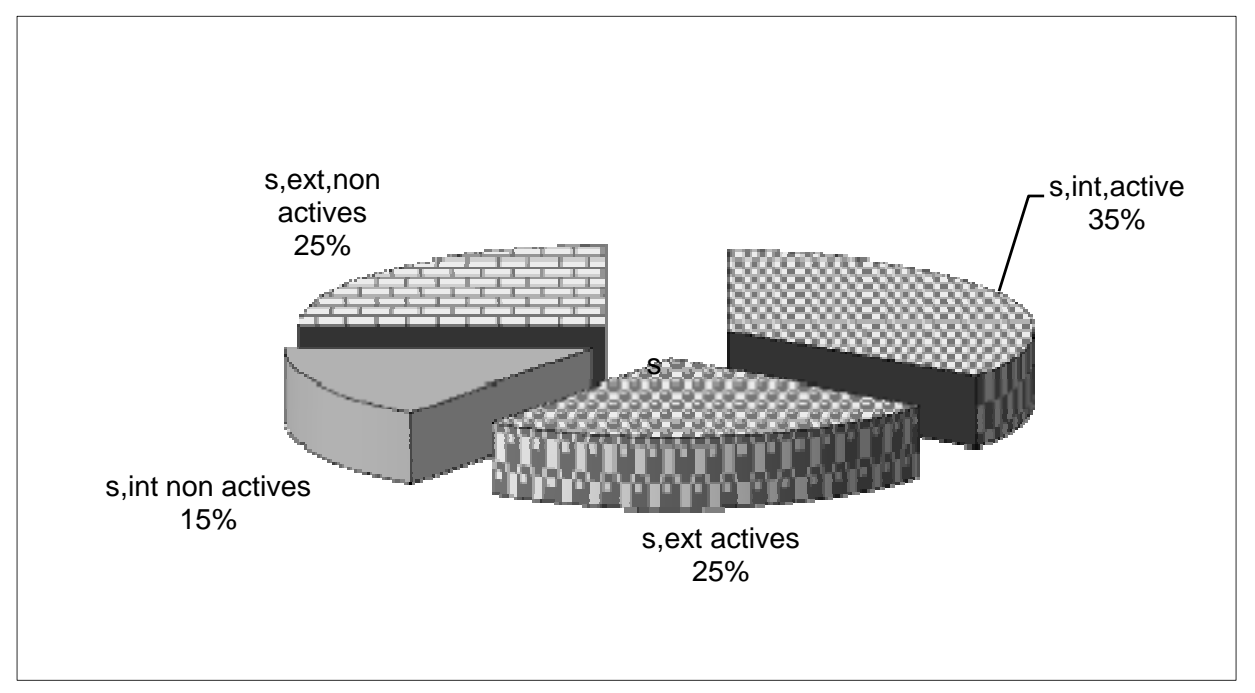

Figure 3: Activité des extraits

S,ext : substances extracellulaires, S,int : substances intracellulaires. 


\section{3-2-1. Effet des extraits aqueux des substances intracellulaires}

Les extraits aqueux des substances intracellulaires n'ont pas présenté une importante activité. Une faible inhibition de la croissance de $S$. marcescens (diamètre de la zone d'inhibition inférieur à $12 \mathrm{~mm}$ ) (Figure 4) est notée pour les extraits aqueux d' O. chlorina(S5), de Phormidium sp. (S9) et de N. muscorum(S10) (Figure 5).

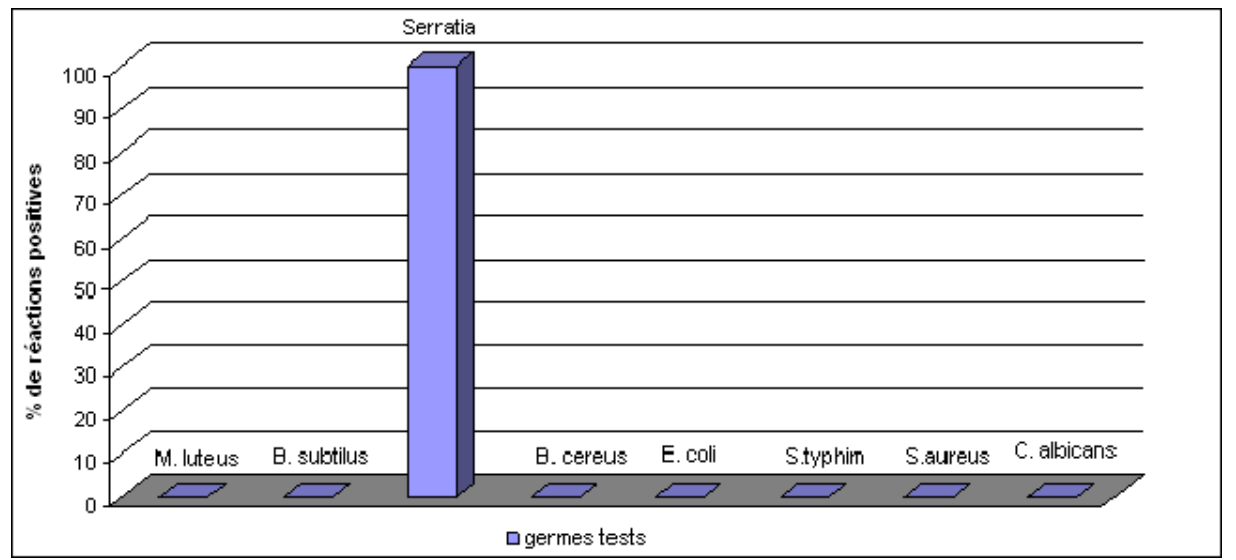

Figure 4 : Sensibilité des germes tests vis-à-vis des extraits aqueux des substances intracellulaires.

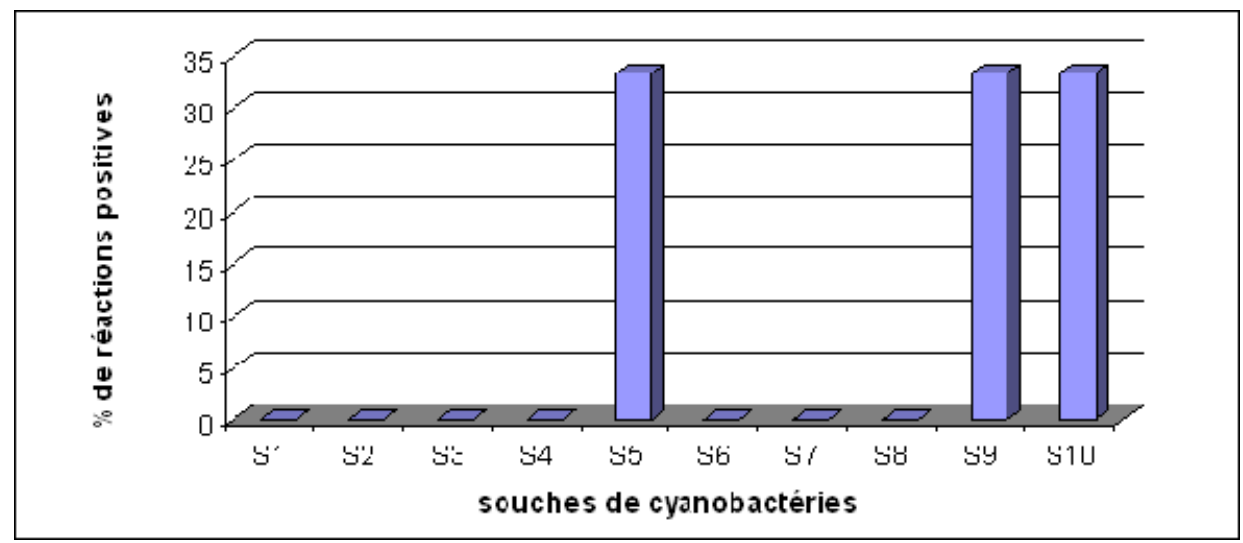

Figure 5: Pourcentage d'activité des extraits aqueux des substances intracellulaires produites par les cyanobactéries.

Les extraits aqueux des cyanobactéries ne présentent généralement pas une importante activité. Un résultat similaire a été signalé dans les travaux de Kreitlow 
et al. [23] et de Cano et al. [12]. Ces derniers auteurs ont trouvé que l'extrait aqueux de Nostoc muscorum est sans effet inhibiteur sur la croissance de $S$. aureus et sur celle de C. albicans. Au contraire, cet extrait stimule la croissance de $S$. aureus de $4,38 \%$.

De même, Kreitlow et al. [23] ont montré que les extraits aqueux de 12 espèces de cyanobactéries appartenant aux genres Anabaena, Oscillatoria, Pseudanabaena, Limnothrix et Synechocystis n'ont pas d'effet sur la croissance des bactéries à Gram négatif ( $E$. coli, $S$. marcescens, et Proteus mirabilis), sur les bactéries à Gram positif (S. aureus, B. subtilis, M. flavus) et sur la levure C. albicans. Mundt et al. [24] ont trouvé que les extraits aqueux de 35 souches de cyanobactéries ne présentent aucun effet sur des bactéries à gram positif ( $B$. subtilis, S. aureus et $M$. flavus), sur la bactérie à Gram négatif ( $E$. col/) et sur la levure $C$. maltosa.

\section{3-2-2. Effet des extraits méthanoliques des substances intracellulaires}

Au cours de notre étude de screening, nous avons révélé que les cyanobactéries peuvent produire des substances à activité antimicrobienne. A l'opposé des extraits aqueux, certains extraits méthanoliques des dix souches de cyanobactéries sont doués d'une activité antibactérienne vis-à-vis de deux germes tests à savoir $M$. Iuteus $(58,33$ $\%$ des RP) et $B$. cereus $(41,67 \%$ des RP), tandis que les autres germes tests sont résistants aux substances intracellulaires de ces cyanobactéries (Figure 6).

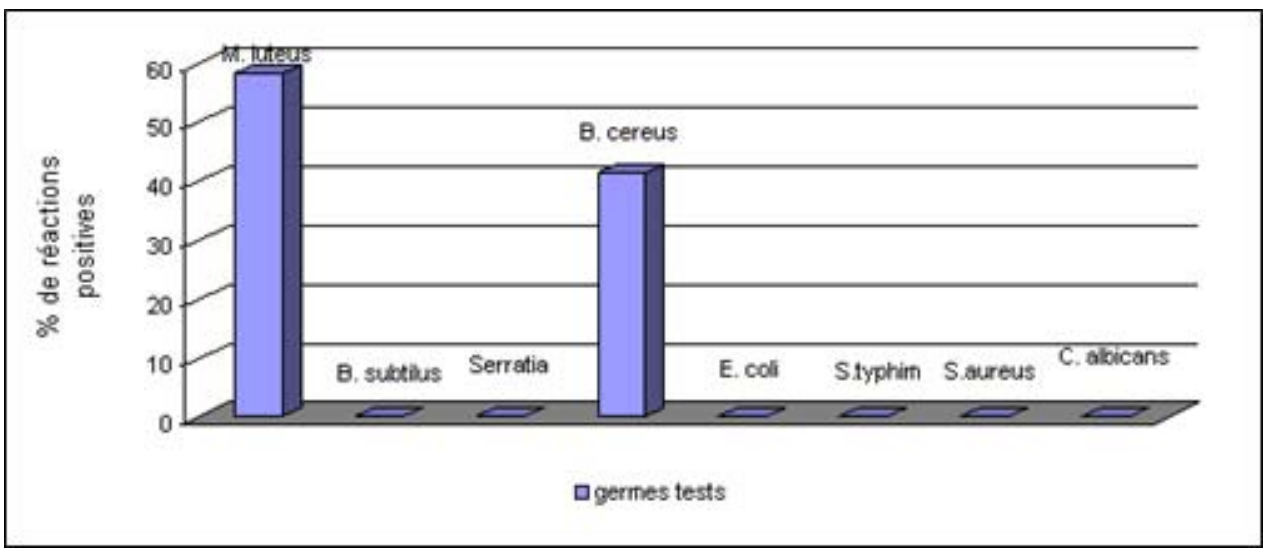

Figure 6 : Sensibilité des germes tests vis-à-vis des extraits méthanoliques des substances intracellulaires.

Les cyanobactéries présentant une activité antibactérienne par leurs extraits méthanoliques sont : Lyngbya sp. (S3)(16,67\% des RP), P. mucicola(S4) (16,67\% des 
RP), O. chlorina (S5) (16,67\% des RP), Phormidium sp. (S9) (16,67\% des RP) et $N$. muscorum (S10) (16,67\% des RP) qui sont actives sur $M$. Iuteus et sur B. cereus alors que 0 . putrida (SI) $(8,33 \%$ des RP) et $M$. aeruginosa ( $(56)$ ( $8,33 \%$ des RP) ont un effet sur M. Iuteus seule (Figure $\Uparrow$.

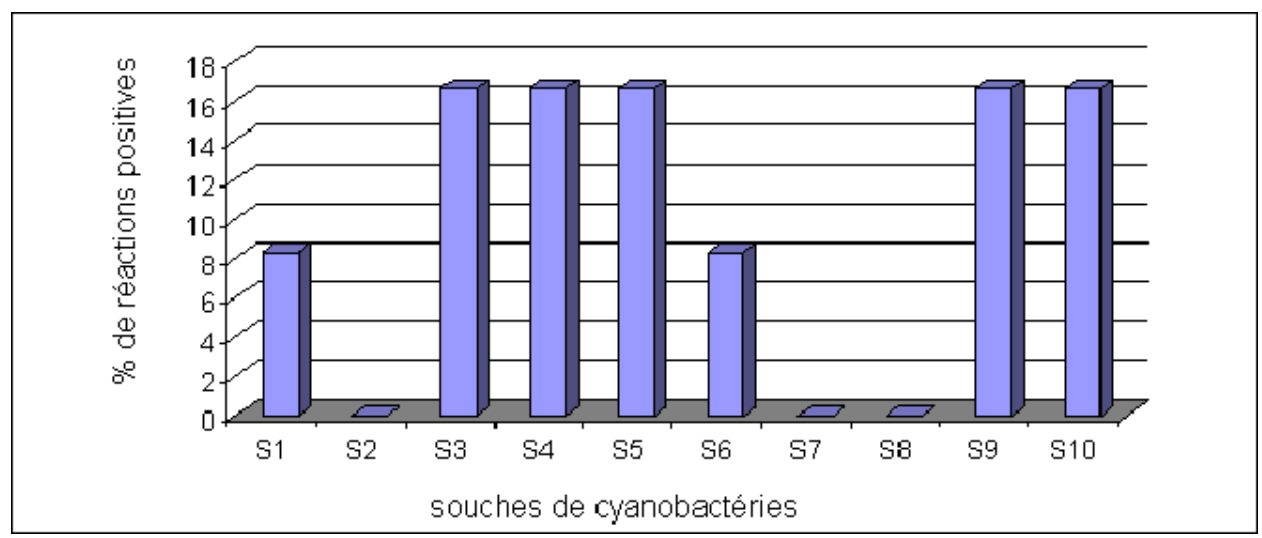

Figure 7 : Pourcentage d'activité des extraits méthanoliques des substances intracellulaires produites par les cyanobactéries.

M. Luteus est le germe test le plus sensible aux extraits méthanoliques des substances produites par les cyanobactéries.

Les travaux de Kreitlow et al. [23] ont montré aussi que Micrococcus est la bactérie la plus affectée par l'extrait méthanolique d'Oscillatoria rubescens 016 . Ces auteurs ont montré que la croissance de $B$. subtilis et $S$. aureus est aussi inhibée par l'extrait méthanolique de cette cyanobactérie, tandis que l'extrait méthanolique d' Oscillatoria tenuis a seulement inhibé la croissance de Micrococcus et de $S$. aureus.

Mundt ef al. [24] ont trouvé de leur part que les extraits méthanoliques d'Anabaena variabilis, de Gloeocapsa caldariorum, de P. catenata et de Limnothrix redekei inhibent la croissance de $B$. subtilis.

Skulberg [20] a rapporté que les extraits méthanoliques de 27 souches de cyanobactéries présentent 31 réactions positives vis-à-vis de $B$. subtilis (6 RP), $B$. cereus(12 RP), Aeromonas hydrophila (12 RP) et E. coli(1 RP).

\section{3-2-3. Effet des substances extracellulaires}

Les substances extracellulaires produites par des cyanobactéries étudiées ont aussi un effet inhibiteur sur les germes tests. En effet, $25 \%$ des produits extracellulaires testés sont actives et arrivent à inhiber la croissance de certains germes tests. Cette 
inhibition est observée chez M. Iuteus, B. subtilis, S. marcescens, B. cereus, E. coli et S. aureus (Figure 8).

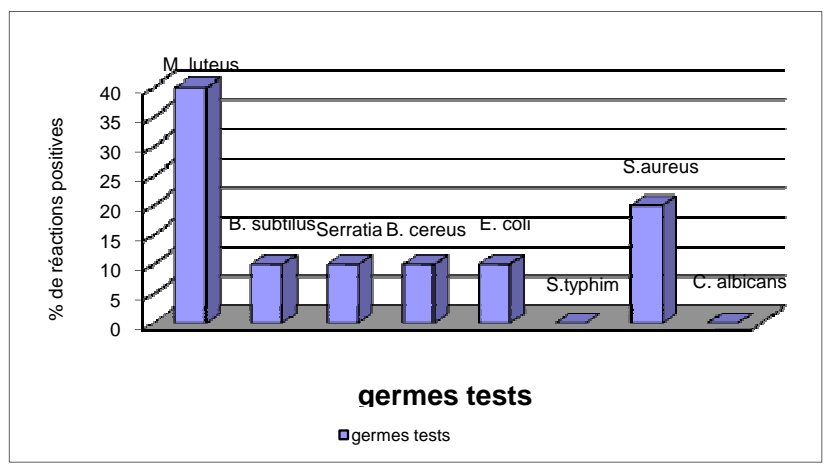

Figure 8 : Sensibilité des germes tests vis-d̀-vis des substances extracellulaires.

0. putrida (SI)est la cyanobactérie la plus active par ses substances extracellulaires. Elle est responsable de $60 \%$ des réactions positives. Elle a un large spectre d'activité car elle arrive à inhiber la croissance de six germes tests: M. luteus, $B$. subtilis, $S$. marcescens, B. cereus, E. coli et $S$. aureus.

Les autres cyanobactéries agissant par les substances extracellulaires sont Lyngbya sp. (S3)(10\% RP), 0. chlorina (S5) (10\% RP) et M. aeruginosa (S6) (10\% RP) qui ont un effet inhibiteur sur M. Iuteus, tandis que Phormidium sp. (S9) (10\% RP) agit sur $S$. aureus seule (Figure 9).

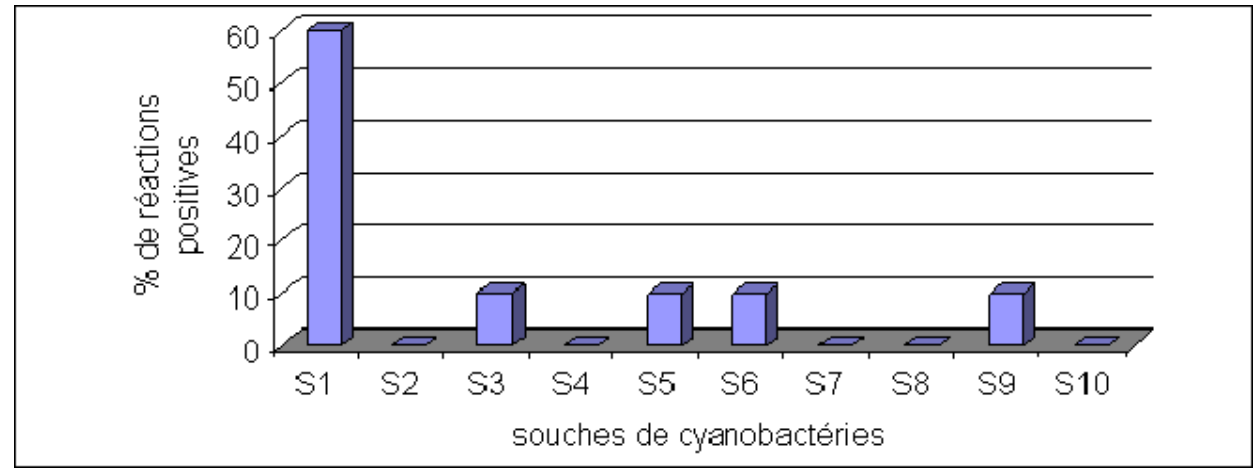

Figure 9: Pourcentage d'activité des substances extracellulaires des cyanobactéries. 
Caire et al. [25] ont trouvé que les substances extracellulaires élaborées par Nodularia punctiforme, Nostoc muscorum, Nostoc piscinale, Anabaena orizae, Tolypothrix tenvis et Scytonema hofmani inhibent la croissance de C. albicans. Alors que les substances excrétées par Microchaete tenera sont sans effet sur cette levure. Ces auteurs ont aussi rapporté que $S$. aureus est inhibée par les substances extracellulaires de $N$. muscorum, $N$. piscinale, $N$. punctiforme et $A$. orizae.

Cependant, Cano et al. [12] ont détecté que les produits extracellulaires de $N$. muscorum n'ont pas d'effet sur la croissance de C. albicans et de S. aureus.

Le screening des substances extracellulaires élaborées par les cyanobactéries, réalisé par Birgit et Ultrich [26] a montré que la croissance d'E. coli est inhibée par les produits d'Oscillatoria limnetica. Les substances excrétées par Anabaena sp., par 0 . subtilissima et par 0 . limnetica ont un effet inhibiteur sur Saccharomyces cervisiae. Alors que $B$. subtilis est résistante aux substances extracellulaires de ces trois cyanobactéries [26].

D'une manière générale, Oscillatoria putrida (SI) est la souche de cyanobactérie la plus active, elle est responsable de $30,43 \%$ des réactions positives (substances intracellulaires et extracellulaires), 0 . chlorina (S5) et Phormidium sp. (S9) sont responsables de 17,39\% des réactions positives pour chacune, Lyngbya sp. (S3) et $N$. muscorum interviennent par $13,04 \%$ pour chacune et finalement $P$. mucicola (S4) et $M$. aeruginosa (S6) par $8,69 \%$ de réactions positives pour chacune. Les trois souches restantes: P. galeata (S2), A. aphanizomenoides (S7) et $L$. sordida (S8) sont inactives (Figure 19).

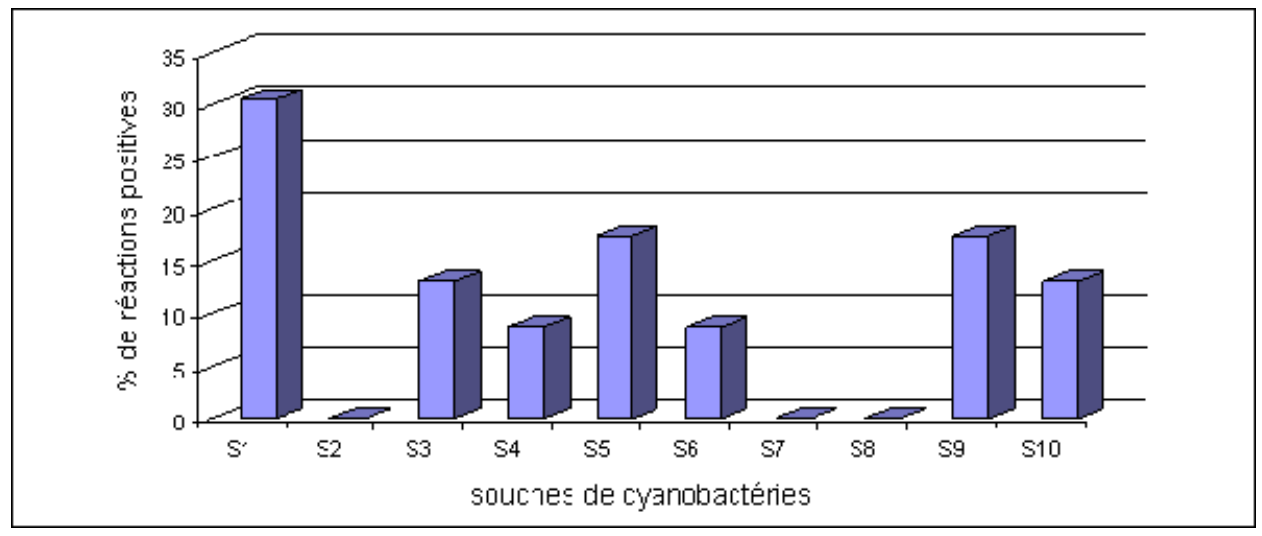

Figure 10: Pourcentage des réactions positives des souches de cyanobactéries.

Ce sont les bactéries à Gram positif qui sont les plus sensibles (Figure II) dont $M$. luteus qui est concernée par $44 \%$ des réactions positives, $B$. cereus par $24 \%, S$. 
aureus par $8 \%$ et $B$. subtilis par $4 \%$, alors que les bactéries à Gram négatif inhibées sont $S$. marcescens (16\% de réactions positives) et $E$. coli $(4 \%$ de réactions positives). $S$. typhimurium et $C$. albicans sont les germes les plus résistants et ne sont inhibés par aucune des cyanobactéries étudiées (Figure 12).

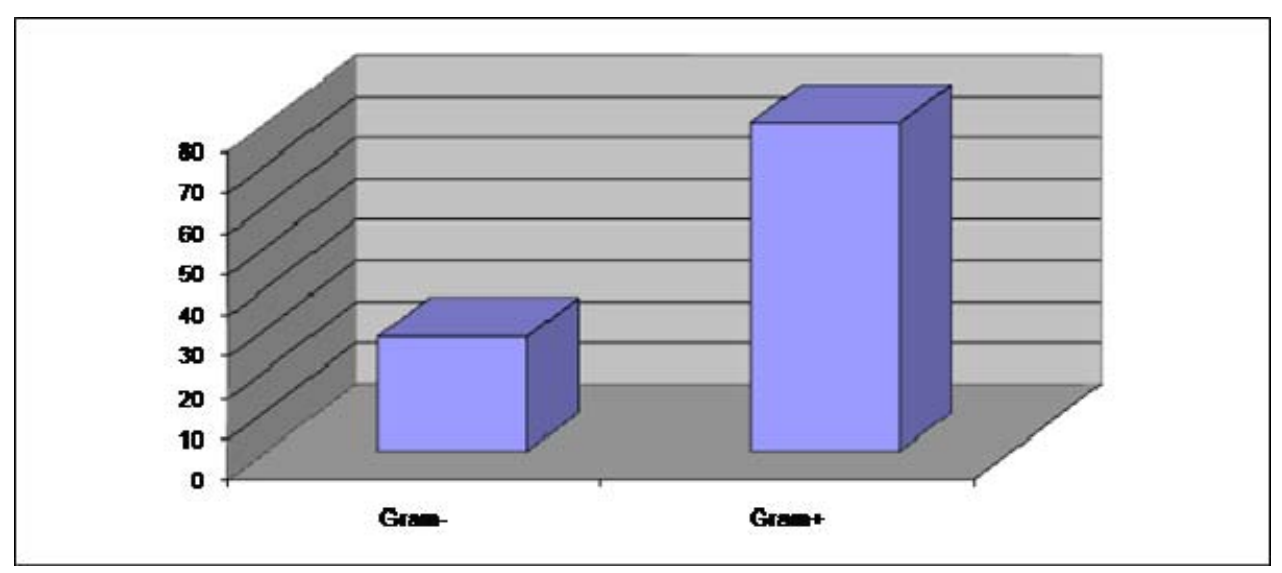

Figure 11: Sensibilité des bactéries Gram+ et des Gram-.

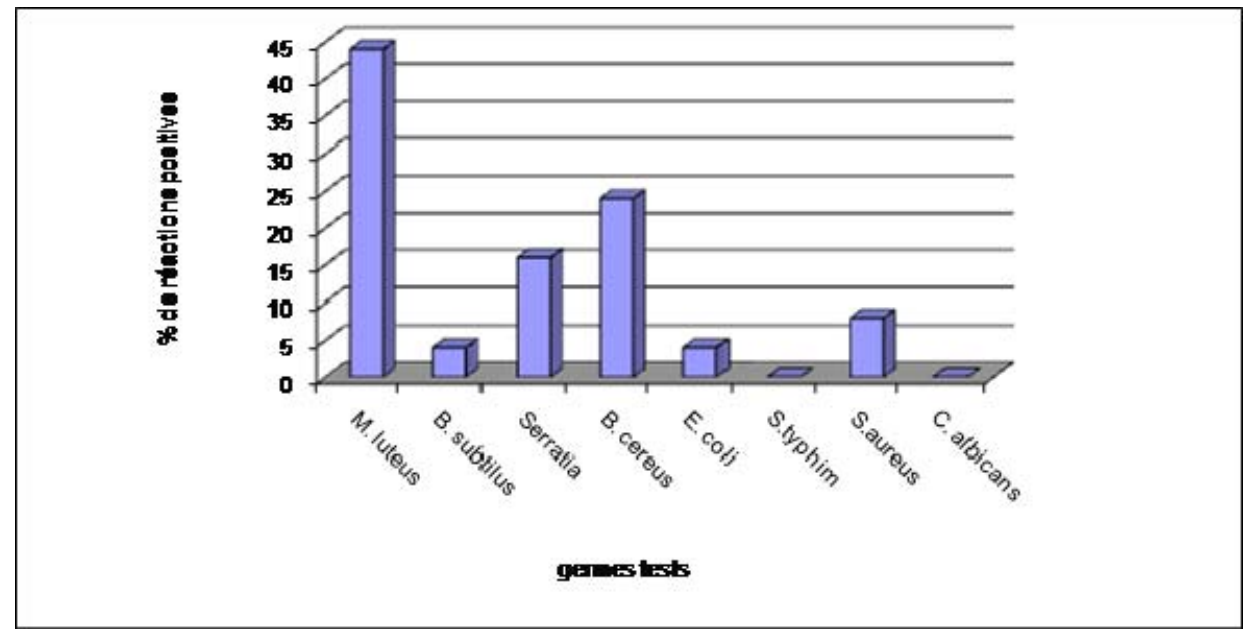

Figure 12: Sensibilité des germes tests.

Les bactéries à Gram positif sont les plus sensibles aux substances élaborées par les cyanobactéries. Ce résultat coïncide avec les observations de Mundt et al. [24] qui ont trouvé que les extraits des cyanobactéries inhibent la croissance des bactéries à 
Gram positif uniquement. Un résultat similaire a été signalé par Kreitlow et al. [23] qui ont rapporté qu'aucun des extraits des cyanobactéries n'a montré d'activité visà-vis des bactéries à Gram négatif.

\section{3-3. Test de toxicité}

Notre screening des extraits cyanobactériens est étendu pour la recherche de substances bioactives toxiques. Pour cela, nous avons testé la toxicité de 7 souches de cyanobactéries: Lyngbya sp. (S3), O. chlorina (S5), P. mucicola (S4), M. aeruginosa (S6), N. muscorum (S10) Phormidium sp. (S9) et 0 . putrida(SI).

Le test souris nous a permis de montrer que 6 souches des cyanobactéries étudiées sont toxiques, il s'agit de Lyngbya sp., O. chlorina, P. mucicola, M. aeruginosa, N. muscorum et Phormidium sp., tandis qu' $O$. putrida est non toxique.

Les $\mathrm{DL}_{50}$ (Dose létale de $50 \%$ de souris testés) de certaines souches sont: $94 \mathrm{mg} / \mathrm{kg}$ pour $P$. mucicola, $31-33 \mathrm{mg} / \mathrm{kg}$ pour $M$. aeruginosa et 15 à $125 \mathrm{mg} / \mathrm{kg}$ pour $N$. muscorum. Dans tous les cas, la $\mathrm{DL}_{50}$ est inférieure à $500 \mathrm{mg} / \mathrm{kg}$ (Norme donnée par Lawton et al. [27] pour juger qu'une espèce est toxique), ce qui montre que ces souches sont potentiellement très toxiques.

Nous notons que la souche Oscillatoria putrida (SI) la plus active par ses composés bioactifs vis-à-vis des bactéries d'intérêt sanitaire est une souche non toxique.

Les cyanobactéries sont aussi connues par la production de cyanotoxines. En effet, elles ont été associées à des intoxications mortelles de troupeaux d'animaux, de poissons et d'oiseaux. Les toxines incriminées sont dans la plus part des cas des hépatotoxines peptidiques ou alcaloïdiques et des neurotoxines alcaloïdiques [2, 28]. Plus d'une soixantaine d'espèces toxinogènes de cyanobactéries ont été identifiées et se rencontrent le plus souvent parmi les genres Microcystis, Oscillatoria, Anabaena et Aphanizomenon [29, 30].

\section{Conclusion}

L'étude des cyanobactéries présente un intérêt important pour pallier à un certain nombre de problèmes rencontrés notamment dans les secteurs alimentaire, industriel, médical, écologique et environnemental. Dans ce contexte, se situe la présente étude dont l'objectif est la réalisation d'un screening de substances bioactives produites par des cyanobactéries isolées de certains écosystèmes aquatiques marocains. 
Sept souches de cyanobactéries parmi les dix étudiées, produisent des substances bioactives qui présentent une activité antibactérienne vis-à-vis de bactéries d'intérêt sanitaire.

Les substances intracellulaires extraites par le méthanol et les substances extracellulaires présentent l'activité la plus importante et ont un effet inhibiteur visà-vis de M. Iuteus, B. subtilis, S. marcescens, B. cereus, E. coli et $S$. aureus. Ces substances à activité antibactérienne pourraient être utilisées en antibiothérapie surtout celles qui ne présentent pas de toxicité.

Notre étude a montré que les souches de $S$. typhimurium et $C$. albicans sont les plus résistantes aux extraits cyanobactériens. Oscillatoria putrida (SI) est la souche de cyanobactérie la plus active. Cette souche présente une attention particulière du fait qu'elle est la souche la plus active vis-à-vis des bactéries étudiées et qu'elle n'est pas toxique.

\section{Remerciements}

Ce travail est financé par la Fondation Internationale pour la Science (ifs projet No F/2826-2).

\section{Références}

[1] - I. Chorus and J. Bartram, "Toxic cyanobacteria in water : A guide to their public health consequences, monitoring and management", WHO publishing, (1999) $416 \mathrm{p}$

[2] - W. W. Carmichael, "The Cyanotoxins", Adv. Bot. Res., (1997) 211-256

[3] - G. A. Codd, L. F. Morrison and J. S. Metcalf, "Cyanobacterial toxins: risk management for health protection", Toxicol. Applied Pharmacol., 203 (2005) 264-272

[4] - I. Stewart, P.J. Schluter and G.R. Shaw, "Cyanobacterial lipopolysaccharides and human health", Environ. Health, 5 (2006) 7-29

[5] - M. R. BOYD, K. R. Gustafson, J. B. Mc MAHON and R. H. Shoemaker (1997), "Discovery of cyanovirin-N, a novel human immunodeficiency virus-inactivating protein that binds viral surface envelope glycoprotein gp 120: potentiel applications to microbicide development", Antimicrob. Agents Chemother, 41 (1997) 1521-1530 
[6] - K. Ishida, H. Matsuda, M. Murakami and K. Yamaguchi, "Micropeptins 478-A and B, plasmid inhibitors from the cyanobacterium Microcystis aeruginosa", J. Nat. Prod. 60 (1997) 184-187

[7] - S. Loya, V. Reshef, E. Mizzrachi, C. Silberstein, Y. Rachamim, S. Carmeli and A. HIZI, "The inhibition of the reverse transcriptase of HIV-I by the natural sulfoglycolipids from cyanobacteria: contribution of different moities to their high potency", J. Nat. Prod., 61 (1998) 891-895

[8] - I. Miyochi, J. Sasner and F. HANEY, "Lipids of cyanobacterium Aphanizomenon flos-aquae and inhibition of Chlorella growth", J. Chemical Ecology, 20 (9) (1994) 2429-2436

[9] - M. Ikawa, J. F. Haney and J. J. SASNER, "Inhibition of Chlorella growth by the lipids of cyanobacterium Microcystis aeruginosa", Hydrobiol., 331 (1996) 167-170

[10] - L. Hagmann and F. Juttner, "Fischerellin A, a novel photosystem II-inhibiting allelochemical of the cyanobacterium Fischerella muscicola with antifungal and herbicidal activity", Tetrahedron Lett., 37 (1996) 6539-6542

[11] - G. M. L. Patterson and C. M. BOLIS, "Fungal cell wall polysaccharides elicit an antifungal secondary metabolite (phytoalexin) in the cyanobacterium Scytonema ocellatum", J. Phycol., 33 (1997) 54-60

[12] - M. S. CANO, G. Z. CAIRE, C. Z. MULE and D. R. Halperin, "Growth control of Staphylococcus aureus and Candida albicans by Nostoc muscorum", Phyton, 46 (1986) 153-156

[13] - K. Oufdou, N. Mezrioui, B. Oudra, M. Barakate and M. LOUDIKI (1998), "Effect of extracellular and endocellular products from cyanobacterium, Synechocystis $s p$. on the growth of some sanitation system bacteria", Arch. Hydrobiol. Suppl. 90: $139-148$

[14] - R. W. Castenholz and J. B. Waterbury, "Oxygenic photosynthetic bacteria". In: Bergey's Manual of Systematic Bacteriology. Vol 3 (Henzyl WR, ed). Williams and Wilkins, Baltimore, MD, (1989) 1710-1727

[15] - L. Geitler, "Cyanophycae". In: kryptogamen flora. Rabenhorst L. (ed). Band 14, Akademisve verlagsgesellchaft, Leipizig, (1932) $1196 \mathrm{p}$

[16] - J. Komarek and K. Anagnostidis, "Modern approach to the classification system of cyanophytes. 3-Oscillatoriales", Arch. Hydrobiol. Suppl., 80 (1986) 327-427

[17] - H. SAS, "Lake restoration by reduction of nutrient loading: expectations, experiences, extrapolations". Academia Verlag Richarz, St. Augustin. Germany, (1989)

[18] - B. OUDRA, M. Loudiki, V. Vasconcelos, B. Sabour, B. Sbiyyaa, K. Oufdou and N. MEZRIOUI "Detection and quantification of microcystins in cyanobacterial strains isolated from lake-reservoirs and ponds in Morroco", Environ. Toxicol., 17 (2002) 32-39 
[19] - M. Loudiki, B. Oudra, B. Sabourd, B. Sbiyyaa, V. Vasconcelos and S. FRANCA, "Taxonomy and geographic distribution of potentiel toxic cyanobacterial strains in Morocco", Annales de Limnologie, 38 (2002) 101-108

[20] - 0 . Skulberg, "Microalgae as a source of bioactive molecules-experience from cyanophyte research", J. Applied Phycology, 12, (2000) 341-348

[21] - B. Sabour, M. Loudiki, B. Oudra, V. Vasconcelos, R. MARTINS, Oubratim S. and B. FAWZI, "Toxicology of a Microcystis ichthyoblabe waterbloom from lake Oued Mellah (Morocco)", Environmental Toxicology, 17(1) (2002) 24-31

[22] - B. OUDRA, "Toxicologie des cyanobactéries (Algues bleues-vertes), cas d'espèces occasionnant des blooms toxiques dans différents milieux aquatiques du Maroc", Thèse de Doctorat d'état Es-Sciences, Université Cadi Ayyad, Faculté des Sciences Semlalia, Marrakech, MAROC, (2001) $118 \mathrm{p}$

[23] - S. Kreitlow, S. Mundt and U. Lindequist, "Cyanobacteria, a potential source of new biologically active substances". Elsevier, 70 (1998) 61-63

[24] - S. Mundt, S. Kreitlow, A. Nowotny and U. Effmert, "Biochemical and pharmacological investigations of selected cyanobacteria", Int. J. Hyg. Env. Health. 203 (2001) 327-334

[25] - G. Z. Caire, M. S. De cano, C. Z. De mulé and D. R. De halperin, "Screening of cyanobacterial bioctive compounds against human pathogens", Phyton, 54 (1) (1993) 59-65

[26] - H. S. Birgit and F. Ulrich, "Extracellular cyanobacterial substances inhibit microbial growth", Int. Microbiol., 3 (2000) 231-234

[27] - L. A. Lawton, C. Edwards and C. M. CODD, "Extraction and high-performance liquid chromatographic method for the determination of microcystins in raw and treated waters", Analysis, 119 (1994) 1525-1530

[28] - I. CHORUS, "Cyanotoxins : occurrence, causes, consequences", Ingrid Chorus Ed., Springer publishing, (2001) $357 \mathrm{p}$

[29] - K. Sivonen and G. JONES, "Cyanobacterial toxins. In toxic cyanobacteria in water: A guide to their public health consequences, monitoring and management", E \& FN Spon, London, (1999) 416

[30]- C. Wiegand and S. Pflugmacher, "Ecotoxicological effects of selected cyanobacterial secondary metabolites", Toxicol. Applied Pharmacol., 203 (2005) 201- 218 